\title{
Interdependence of histamine and methylhistamine kinetics: modelling and simulation approach
}

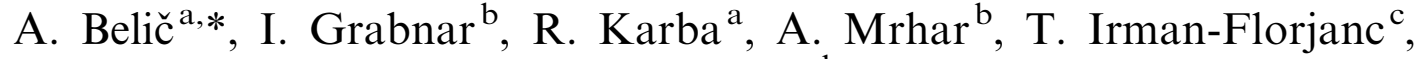 \\ S. Primožič ${ }^{b}$ \\ ${ }^{a}$ Faculty of Electrical Engineering, University of Ljubljana, Tržaška 25, 1111 Ljubljana, Slovenia \\ ${ }^{\mathrm{b}}$ Faculty of Pharmacy, University of Ljubljana, Ljubljana, Slovenia \\ ${ }^{\mathrm{c}}$ Department of Pharmacology, Medical Faculty, University of Ljubljana, Ljubljana, Slovenia
}

Received 10 December 1998; accepted 28 July 1999

\begin{abstract}
In the article a model of histamine kinetics is described. A motivation of this project was to investigate the hypothesis that methylhistamine may be a marker of histamine appearance in plasma. A model has been made to support the hypothesis. Since metabolic and transport pathways of histamine and methylhistamine are complex and not very well known, the relationship between histamine and methylhistamine should be elucidated by mathematical modelling. From experimental data and the information in the literature, a nonlinear and time-varying four-compartment model is proposed. Extensive release of histamine from mast cells when methylhistamine is injected, is modelled as histamine to methylhistamine ratio control loop. (C) 1999 Elsevier Science Ltd. All rights reserved.
\end{abstract}

Keywords: Histamine; Methylhistamine; Pharmacokinetics; Control loop; Modelling; Compartment models

\section{Introduction}

Histamine is an endogenous amine, present in humans, animals and plants. It is known as a mediator in physiological processes and is involved in many pathological processes. During inflammatory or allergic reactions it is released from stores in mast cells and has an important role in adverse reactions. When released, it is rapidly inactivated by metabolic processes $[1,2]$

\footnotetext{
* Corresponding author. Tel.: + 386-61-1768-417; fax.: + 386-61-1264-631.

E-mail address: ales.belic@fe.uni-lj.si (A. Belič)
}

0010-4825/99/\$ - see front matter (C) 1999 Elsevier Science Ltd. All rights reserved.

PII: S0010-4825(99)00017-7 
and therefore is difficult to measure the rapid changes of histamine concentrations in body fluids.

Efforts are made to find a marker for histamine appearance in the body. It is found in the literature that its metabolite, methylhistamine (tele-methylhistamine, $N$-methylhistamine) may be one of them. It has been reported that histamine- $N$-methyltransferase, one of the two important enzymes (the second is diamine oxidase) involved in transformation of histamine, was shown to be stimulated in the cerebral cortex of adult rats by chronic stress [3]. The elimination half-life of methylhistamine $(\mathrm{M})$ in plasma of the rat is about ten times longer than of histamine (Hi) $\left(t_{1 / 2}(\mathrm{M})=43 \mathrm{~min}, t_{1 / 2}(\mathrm{Hi})=3.5 \mathrm{~min}\right)$. There is also a threefold methylhistamine plasma to whole blood concentration ratio compared to histamine [1]. Since metabolic and transport pathways of histamine and methylhistamine are complex and not very well known, the relationship between levels of the two substances in plasma may be elucidated by mathematical modelling, to support a hypothesis that methylhistamine can be a marker of histamine appearance in plasma.

Generally, to show that the interdependence between the two substances is substantial, the model must be able to explain the available experimental data by taking into account metabolic and transport mechanisms involving the two substances.

Mathematical modelling [4], starting from basic principles of chemical and enzymatic reactions, is not possible. Firstly, metabolism of histamine and methylhistamine is not completely understood and secondly, the modelling objective does not require such a complex mathematical model. Therefore, we used a physiologically based compartment model [5-7]. The basic structure of the compartment model is derived from known pharmacokinetic characteristics of the substances. Parameters of the model were estimated by curve fitting procedures [8]. Validation of the model is performed by evaluating the quality of the fit of model response and measured data and by analysing model parameters.

A physiologically based compartment model is an optimal solution of the problem, since both experimental data and theoretical knowledge of the process can be combined.

Information about histamine and methylhistamine kinetics was taken from the literature [911] and the experiments performed within this study. The model was developed according to the information and the fit to the plasma concentration data collected from rats.

\section{Methods}

\subsection{Experiments}

The levels of histamine and methylhistamine were measured in rats in two separate groups of experiments. In the first group, rats were treated by histamine intravenously. In the second group, however, methylhistamine was administered intravenously. Both substances were administered by IV bolus injection. In both groups of the experiments histamine and methylhistamine were assayed simultaneously in the plasma samples by HPLC method [1]. The dose of histamine was $14.65 \mu \mathrm{g}$ and dose of methylhistamine was $15.66 \mu \mathrm{g}$. The samples were taken 2, 4, 6, 10, 15, 20, 30 and $60 \mathrm{~min}$ after the histamine dose and 2, 3, 4, 5, 10, 15, 20, 30, 40, 50 and $60 \mathrm{~min}$ after methylhistamine dose. Only up to two $2 \mathrm{ml}$ samples were taken from 
each rat, to prevent hemoragic shock. There were two to nine data points at each sample time, therefore, average value at each sample time was calculated, to obtain plasma profiles of both amines.

\subsection{Modelling}

Three different tools were used in model development: a stripping method [5-7], an analogue-hybrid computer and MATLAB with SIMULINK, general numerical analysis software.

Program package PCNONLIN was used for stripping. Linear regression to log transformed data was used to determine exponential series best fitting the data. Stripping was applied separately for each amine. Rate constants and volumes of distribution were derived from the series for the assumed histamine and methylhistamine model structure. These parameters were a starting point for modelling and fitting procedures on analogue-hybrid and digital computer.

On analogue-hybrid computer model parameters were estimated by manually changing parameter values and observing optical quality of fit. MATLAB with SIMULINK was used as an alternative to analogue-hybrid computer. The combination of both tools provided better information on model quality. However simpler application of optimzation methods for parameter estimation and more objective measure of fit were major advances of digital approach. Model parameters were estimated by simplex optimization method. The objective of optimization was to minimize Integral of Squared Error (ISE) of model response and measured data. Since the curves were represented digitally i.e. as points, ISE was calculated as sum of squared errors. For the first model (1) linear interpolation was used between measured points and ISE indicated distance between model response and interpolated curve. For the second (2) and the third (3) model no interpolation was used and ISE was calculated as a sum of squared errors at times of measurements. For model 3 the value of ISE for the first experiment was added to the value of ISE for the second experiment, since the model was fitted to the data of both experiments simultaneously. Therefore, comparison of the ISE values between models provides only small amount of information on the quality of fit. Different measures of fit were used to reduce local minima problems for the optimization method.

Compartment models were used to model the kinetics. Model structure was composed from physiological background of the process and stripping results and was modified according to parameter estimation results. Parameter values close to zero indicated small relevance of the parameter on modelled kinetics and model could be simplified by omitting the parameter. The model structure was also modified if the model could not fit the concentration profiles acceptably. Modifications to the model were made only, if physiological explanation could also be found.

\section{Results}

Pharmacokinetic modelling often begins with a linear compartment model [5-7], in spite of the fact that measured data may show nonlinear characteristics. This way basic information for model development can be extracted from the measurements. 
Since linear regression of $\log$ transformed data rendered correlation parameters greater than 0.900 for both amines, a linear model was first proposed and examined. It has been known from our previous studies [1,2] and the results obtained by the stripping method within this study that a two-compartment model describes plasma profiles of both amines satisfactorily. In order to investigate the hypothesis that methylhistamine is the marker of the plasma histamine level changes, the two two-compartment models have to be combined into a four-compartment model (model 1) with methylation as binding mechanism (Fig. 1). At this point, i.e. when trying to couple the two two-compartment models into one four-compartment model, the method of stripping failed. Therefore the model was fitted to the measured data on the analogue-hybrid parallel processor EAI 2000. After that, the rate constants obtained by analogue-hybrid simulation were used as initial estimates for the fitting procedures on digital computer (MATLAB with SIMULINK), since randomly generated initial estimates do not provide useful results in simultaneous fitting procedures.

Rate constants determined by the two different tools are quite different which is not

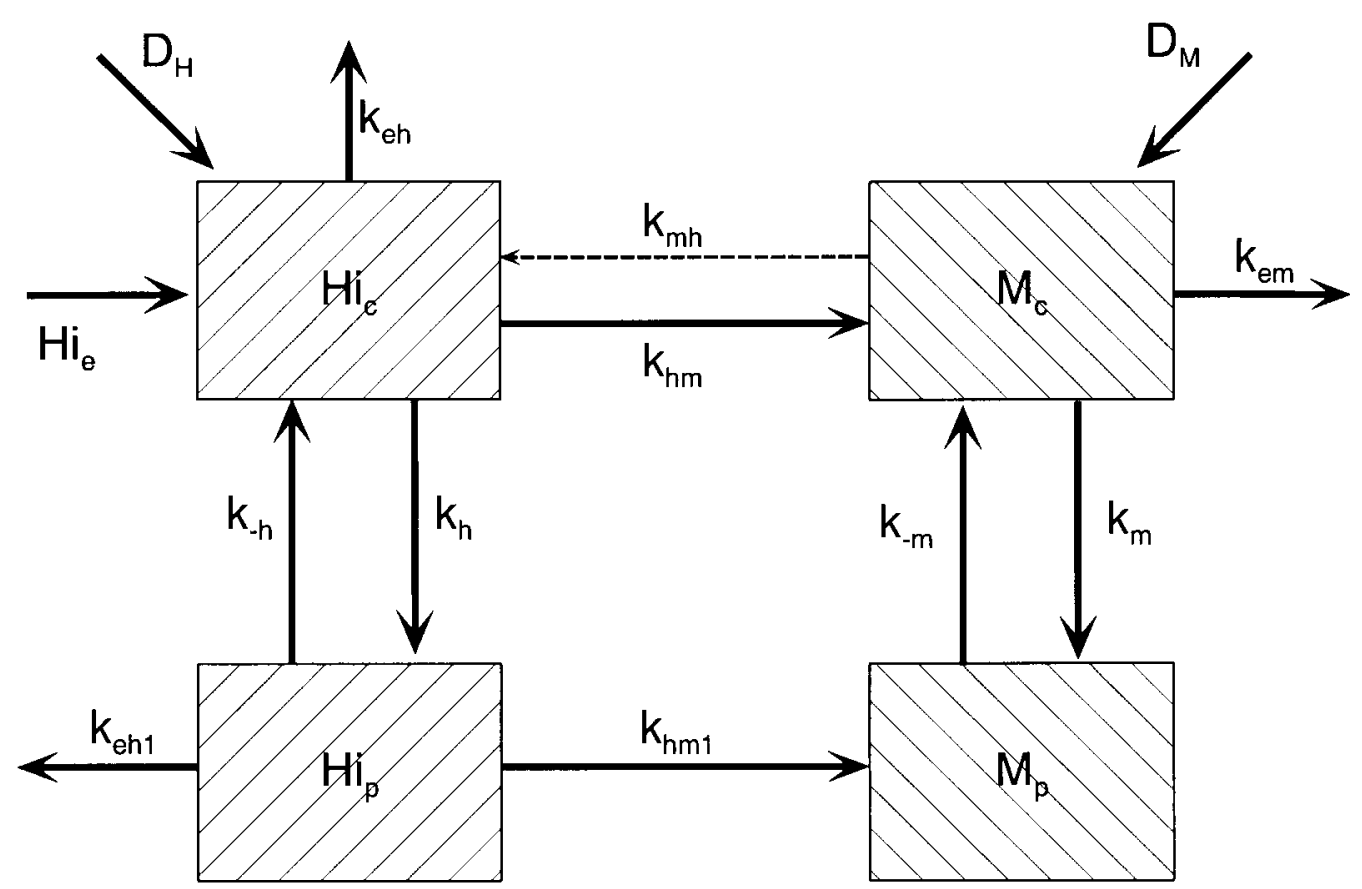

Fig. 1. Model 1. Linear model of histamine kinetics. The labels have the following meaning: $D_{H}=$ histamine injection, $D_{M}=$ methylhistamine injection, $H i_{c}=$ quantities in central compartment for histamine (plasma and well perfused organs), $H i_{p}=$ quantities in peripheral compartments for histamine (the rest of the tissues, not included in the central compartment), $M_{c}=$ quantities in central compartment for methylhistamine (plasma and well perfused organs), $M p=$ quantities in peripheral compartment for methylhistamine (the rest of the tissues, not included in central compartment), $H i_{e}=$ endogenous histamine, $k_{e h}, k_{e h 1}, k_{e m}=$ metabolic/elimination rates for both substances, $k_{h}, k_{-h}, k_{m}, k_{-m}=$ exchange rates between central and peripheral compartment for both substances, $k_{h m}, k_{h m 1}=$ rate constants representing methylation in central and peripheral compartment, $k_{m h}=$ rate constant representing demethylation in central compartment. Note that demethylation is presented by a broken line, since it is present in the model only when methylhistamine is injected. The thick lines represent elimination and methylation. 
surprising, since the model is not uniquely identifiable [12,13] and therefore more sets of rate constants provide acceptable fitting to the experimental data. To support the interdependence of the two substances, the simplest possible model is needed, therefore model parameters have more or less only a qualitative relation to real physiological processes. However, considering the model and the modelling objective, only qualitative interpretation of model parameters is relevant, therefore, exact estimation of parameters is not necessary and nonidentifiability of the model is not a problem [14].

To provide better physiological information in all figures, concentration profiles are shown. Concentrations in central compartment represent the quantities in the central compartment divided by the volume of distribution. The latter is for histamine $0.177 \quad 1$ and for methylhistamine $0.167 \mathrm{l}$. Only simulation results obtained by MATLAB-SIMULINK are presented. The results of simulations are presented in Figs. 2-5. Figure 2 shows a good quality of fit for experiment where histamine is injected. In Fig. 3 the model with the same parameter values is used to predict histamine levels for the experiment when methylhistamine is injected. It can clearly be seen that level rebound cannot be fit without the presence of demethylation in the linear model. The model was then fitted to methylhistamine injection data and histamine injection data was predicted (Fig. 4). The results show that it is impossible to describe the two situations with only one set of parameter values for a linear model. Therefore two versions of the model 1 (Fig. 1) with the same structure and different parameter values are needed, one for each type of experiment. Model 1a describes the situation when histamine is injected and model $1 \mathrm{~b}$ describes the situation when methylhistamine is injected.

In the case of injected histamine, the model complies with known physiological characteristics of the process. On the contrary, when methylhistamine is injected, the presence of demethylation (Fig. 3) is indicated by the model. As this is the only way that the model can fit the profiles, this process has been taken into consideration in spite of the fact that $N$ demethylation on imidazole ring of histamine is not expected to occur [15]. One of the reasons for such difference in complexity of the processes in the two groups of experiments may be the
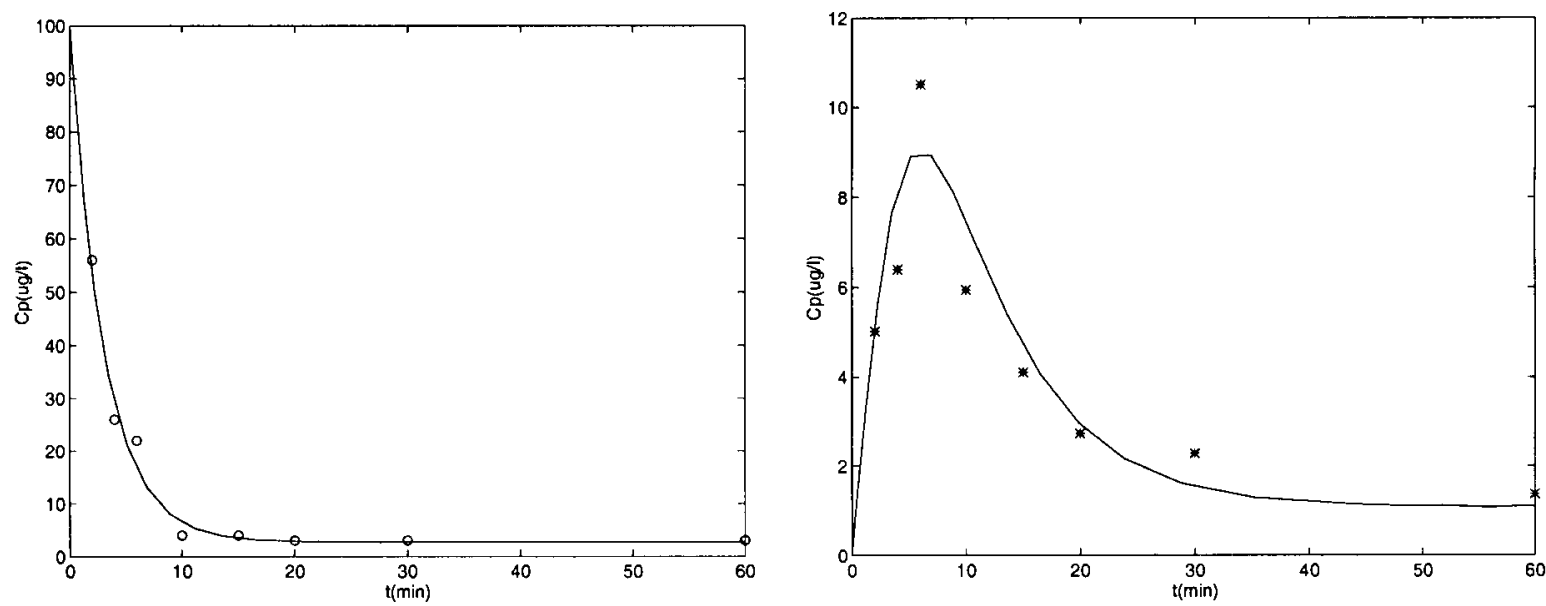

Fig. 2. The model la responses (lines) compared to measured data $(\bigcirc=$ histamine, $*=$ methylhistamine) after histamine intravenous administration. 


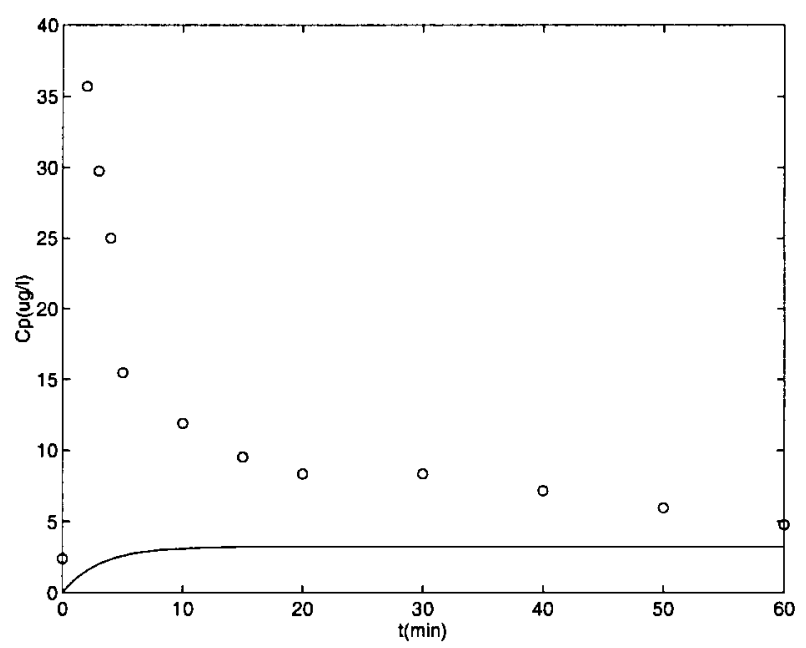

Fig. 3. The model 1a response (line) compared to measured data $(\bigcirc=$ histamine). Model 1a is used for predicting the levels of histamine after methylhistamine intravenous administration.

fact that an injection of histamine is closer to natural processes, compared to methylhistamine injection. The injection of histamine may mimic an extensive histamine release from mast cells in the body whereas the injection of methylhistamine does not simulate a known natural process.

Model responses of the two models (Fig. 1) are shown in Fig. 5. As can be seen from Fig. 5 the quality of fit is acceptable, however, only when demethylation is included in the model.

The linear model fitted the data reasonably well. Unfortunately it took two models to describe histamine kinetics of the two experiments. Moreover, it includes the possibility of demethylation which is questionable as noted a while ago. Therefore, another mechanism should be introduced in the model to comply better with biological backgrounds. This lead to
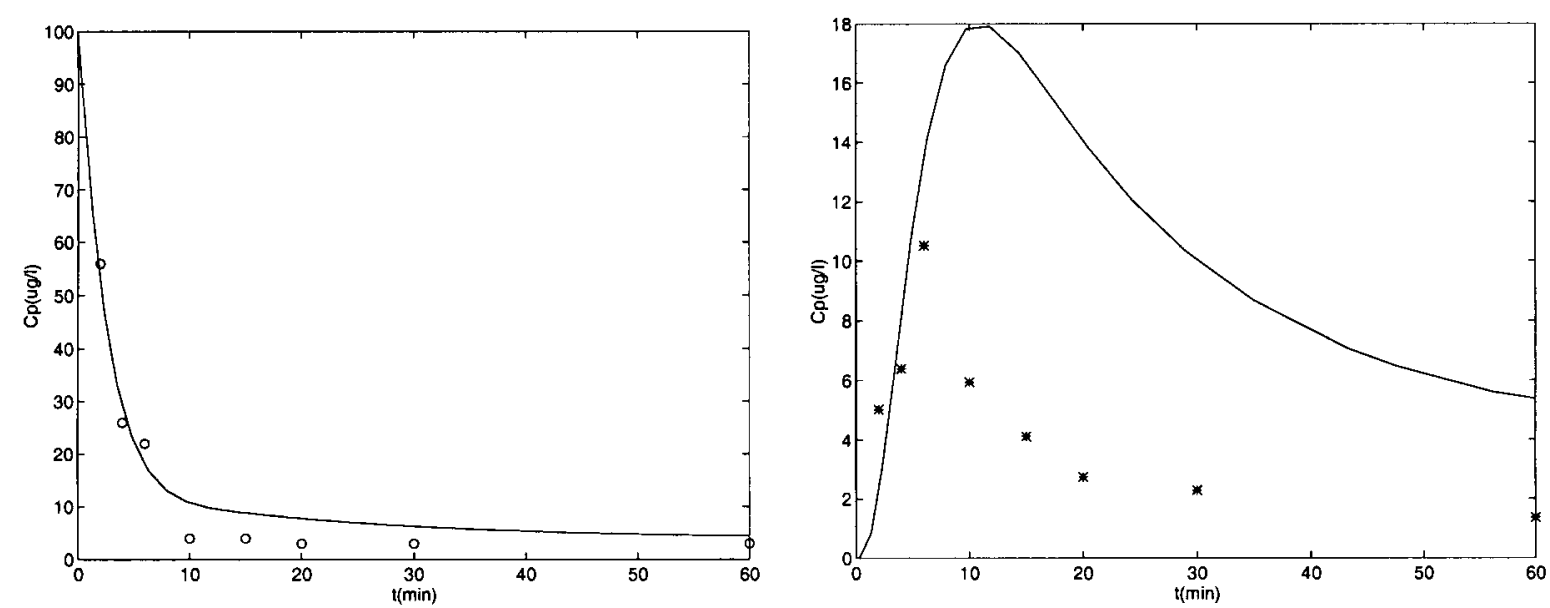

Fig. 4. The responses of the model $1 b$ (line) compared to measured data $(\bigcirc=$ histamine, $*=$ methylhistamine). Model $1 \mathrm{~b}$ is used for prediction of levels after histamine intravenous administration. 

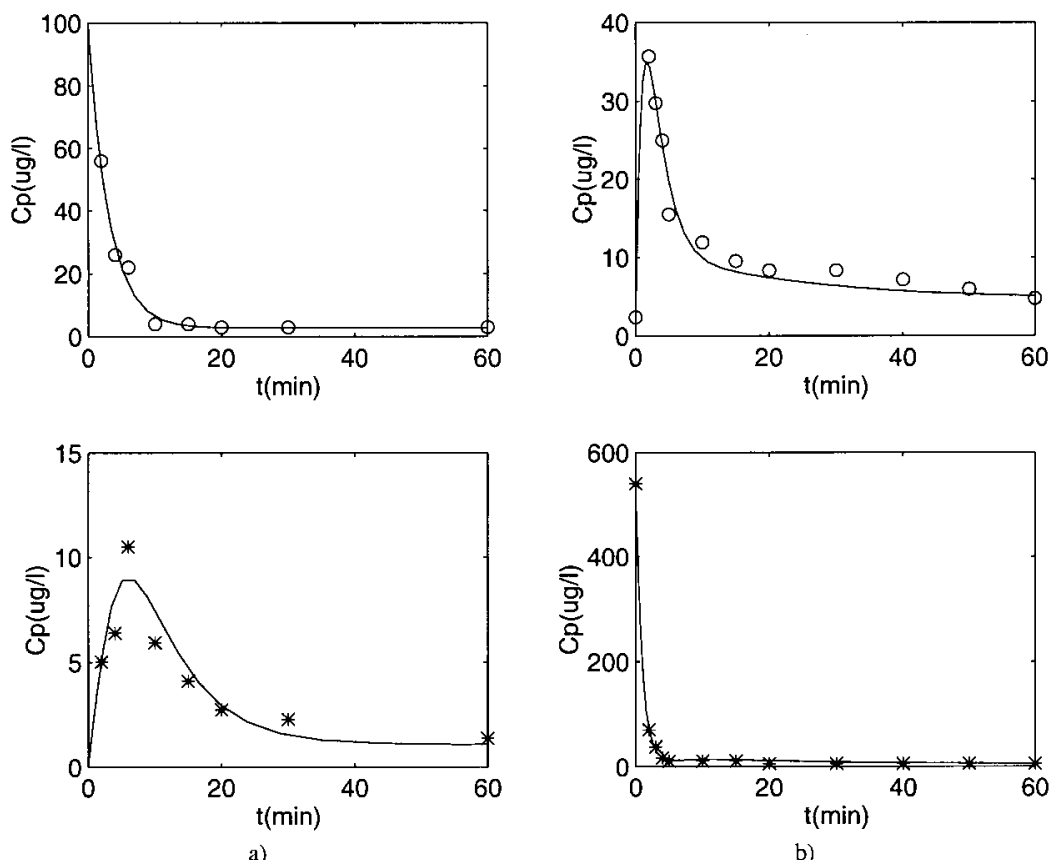

Fig. 5. Responses of the models $1 \mathrm{a}$ and $1 \mathrm{~b}$ (lines) compared to measured data $(\bigcirc=$ histamine, $*=$ methylhistamine $)$ (a) model la after histamine intravenous administration, (b) model $1 \mathrm{~b}$ after methylhistamine intravenous administration (demethylation included in the model!).

the conclusion that linear models are too simple to describe histamine kinetics satisfactorily. Therefore a nonlinear and time varying model was developed.

We then went on towards the unification of the two models into a single four-compartment model, i.e. a model able to describe histamine pharmacokinetics with only one set of parameters irrespective of the injected amine. The structure of the model remains once again the same for both experiments. Therefore, the most transparent solution is to model the difference in rate constants between the two groups of experiments as quantity dependent step functions (Eq. (1)).

$$
k_{h m}\left(M_{c}\right)= \begin{cases}k_{h m}^{\prime} ; & M_{c}<C \\ k_{h m}^{\prime \prime} ; & M_{c} \geq C\end{cases}
$$

Since the linear model fits the data reasonably well and this finding is in accordance also with the results obtained by stripping, i.e. linear and not initially convex and then linear relationship of log transformed data for both amines, it can be concluded that in the observed range of histamine and methylhistamine levels no saturation occurs. Therefore, MichaelisMenten kinetics could not be applied to describe methylation and other metabolic transformations of histamine and methylhistamine and as a way out, step functions were used. They provide more simple and transparent model, since it is piece-wise linear.

Within this attempt of single model generation, methylation and elimination rates were modelled as quantity dependent. The rate constants $k_{h m}, k_{h m 1}, k_{e m}, k_{e h}$ and $k_{e h 1}$ should be viewed rather as metabolic than elimination constants, characterizing histamine $N$-methylation 
in central and peripheral compartments $\left(k_{h m}, k_{h m 1}\right)$ which is catalized by $N$-methyltransferase, histamine oxidative deamination $\left(k_{e h}, k_{e h 1}\right)$, which is catalized by diamine oxidase and $N$ methylhistamine oxidative deamination $\left(k_{e m}\right)$ which is catalized by monoamine oxidase. Since it has been shown that the above mentioned enzymes are extended over a wide range of tissues and as a result only $1-4 \%$ of unchanged histamine is excreted to urine [16], it is reasonable to include into the model metabolic (elimination) pathways for both amines also from peripheral compartments. The rate constant $k_{-h}$ is omitted since fitting procedures showed that it has negligible influence on the model. The same is valid also for the constant characterizing metabolism/elimination of methylhistamine from peripheral compartment.

The model, referred to as model 2, (Fig. 6) becomes too complex for fitting procedures on an analogue-hybrid computer. Therefore, only MATLAB with SIMULINK was used. However, the initial estimates of parameter values in the fitting procedures were still the ones defined by the curve fitting procedures on the analogue-hybrid computer for models $1 \mathrm{a}$ and $1 \mathrm{~b}$.

Model 2 provides no significantly better fit but explains some mechanisms better and confirms the others. The model is flexible enough and all physiological information about histamine and methylhistamine kinetics can be considered.

The thorough discussion about demethylation mechanism results in the conclusion that it is highly questionable and should be omitted from the model. Instead of demethylation, time dependent endogenous histamine release from mast cells is introduced to explain the sudden rise of histamine plasma levels when methylhistamine was injected intravenously (Fig. 7).

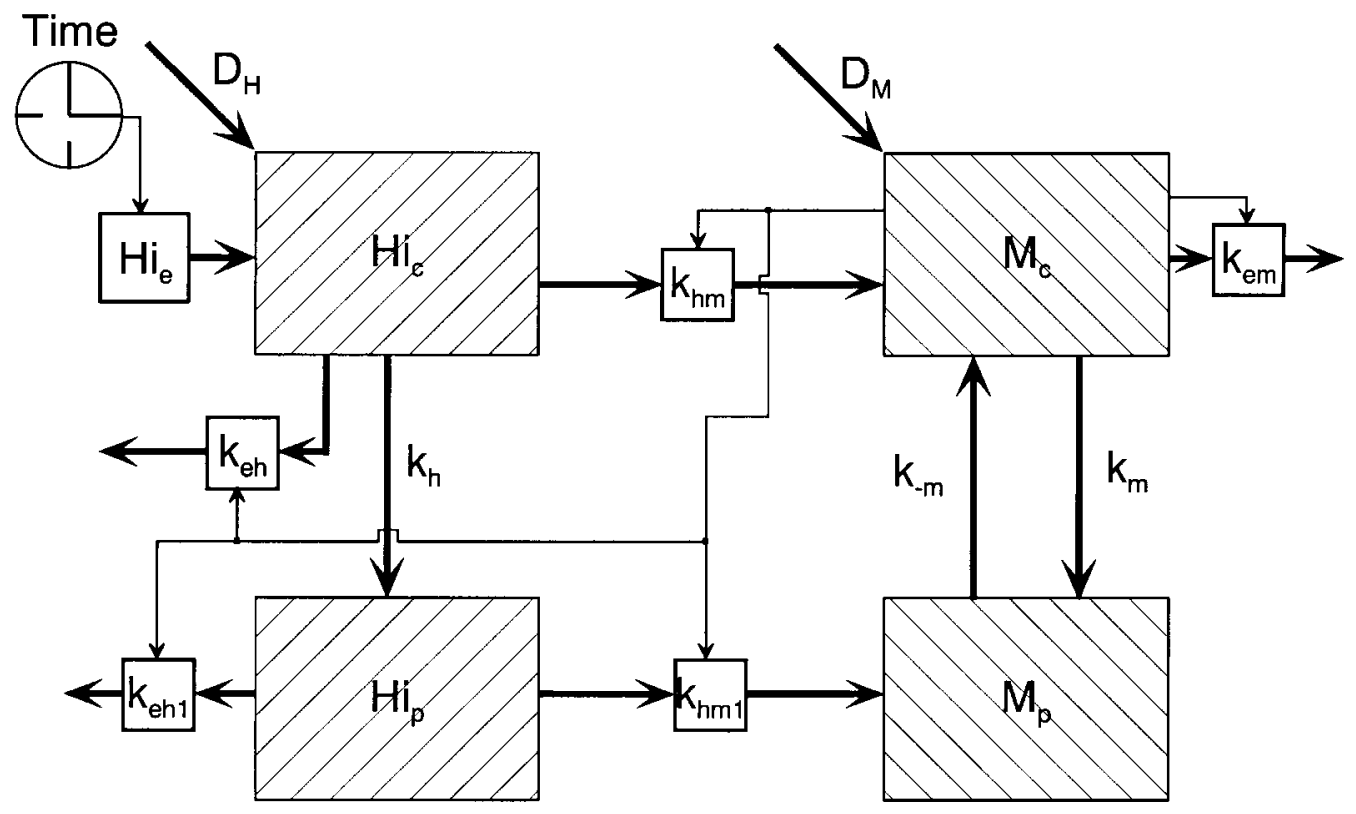

Fig. 6. Model 2 (a and b). Nonlinear model with discrete changes of parameters. The variable parameters are drawn as blocks with thin lines denoting the causes of the changes (parameters connected to an $M$ compartment depend on the quantity in the compartment). Label $\mathrm{Hi}_{e}$, representing the release of histamine from mast cells, is modelled with the time dependent function when methylhistamine is injected (model 2b) and has a constant value when histamine is administered (model $2 \mathrm{a}$ ). 

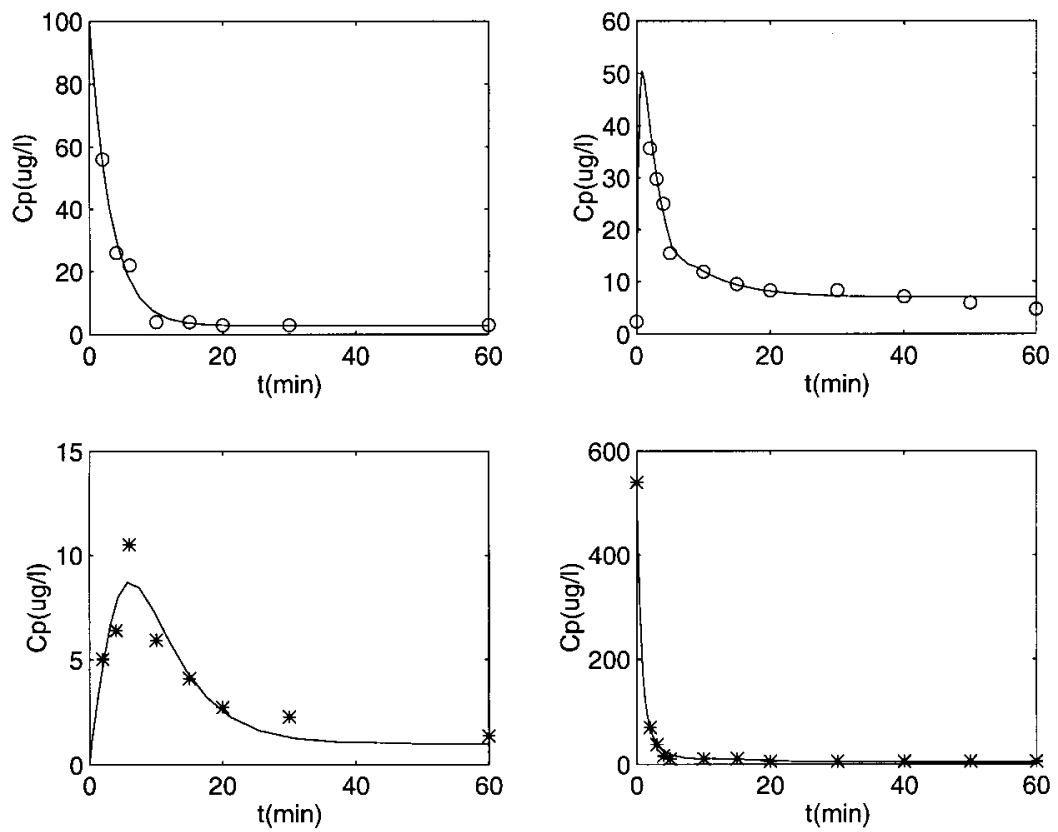

a)

b)

Fig. 7. Responses of the models $2 \mathrm{a}$ and $2 \mathrm{~b}$ (lines) compared to measured data $(\bigcirc=$ histamine, $*=$ methylhistamine $)$ (a) model $2 \mathrm{a}$ after histamine intravenous administration, (b) model $2 \mathrm{~b}$ after methylhistamine intravenous administration (demethylation omitted from the model!).

It has been shown previously in [17] that mast cells are capable of taking up methylhistamine in a time- and dose-dependent manner. It is assumed that the accumulation of methylhistamine in mast cells is due to a simple diffusion process. Moreover, methylhistamine competes with the

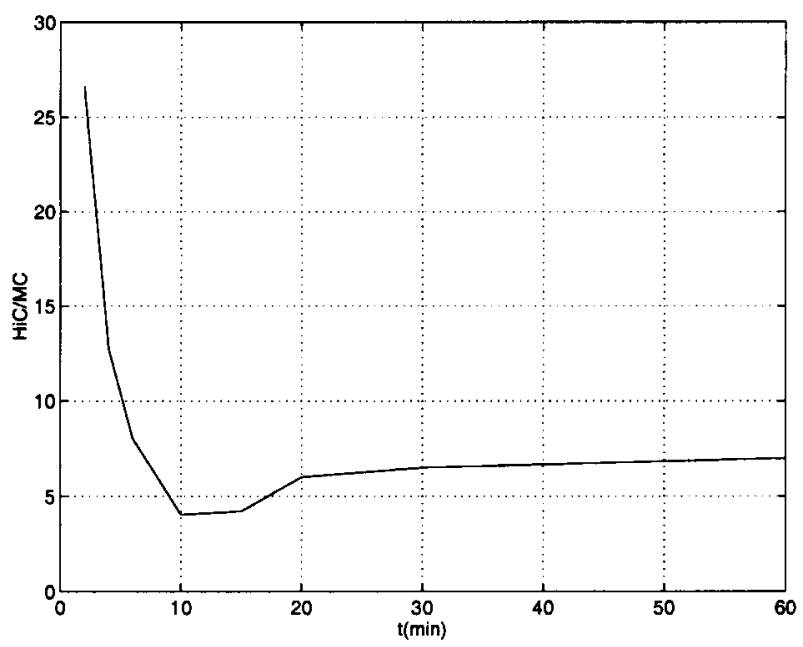

Fig. 8. Average histamine to methylhistamine ratio tends to a constant value. After perturbation of the system it settles at around 7 (measured data). 
same binding sites in mast cells as histamine and as a result, displacement of histamine from mast cells occurs. This comes out as a "burst effect" and the greater part of histamine is released in plasma in few minutes after methylhistamine IV bolus injection.

Two models 2 are still needed, however, the difference between the models is reduced to a rapid histamine release submodel. Model 2a describes the situation when histamine is injected and model $2 \mathrm{~b}$ describes the situation when methylhistamine is injected. The rapid histamine release submodel is present only in the model $2 \mathrm{~b}$.

The time dependent function of endogenous histamine is discussed later. All parameters in Fig. 6 have the same meaning as their corresponding rate constants in Fig. 1.

The time dependent function of extensive histamine release from mast cells represents a problem for model unification. The rebound of histamine levels when methylhistamine is injected indicates that there are significant influences of methylhistamine levels on histamine release from mast cells. The kinetics of both substances may reflect some kind of control mechanisms, since they are endogenous substances. Therefore in order to combine the two separate models $2 \mathrm{a}$ and $2 \mathrm{~b}$, an additional control mechanism has to be introduced. Careful examination of the data shows that the ratio of histamine to methylhistamine levels converges to an approximately constant value after the perturbation (Fig. 8) in both experiments.

These facts lead to the presumption, that there may be some ratio control mechanism involved in the process. The idea is that the histamine to methylhistamine ratio in plasma may be controlled by histamine release from mast cells [17]. The input and the output of the

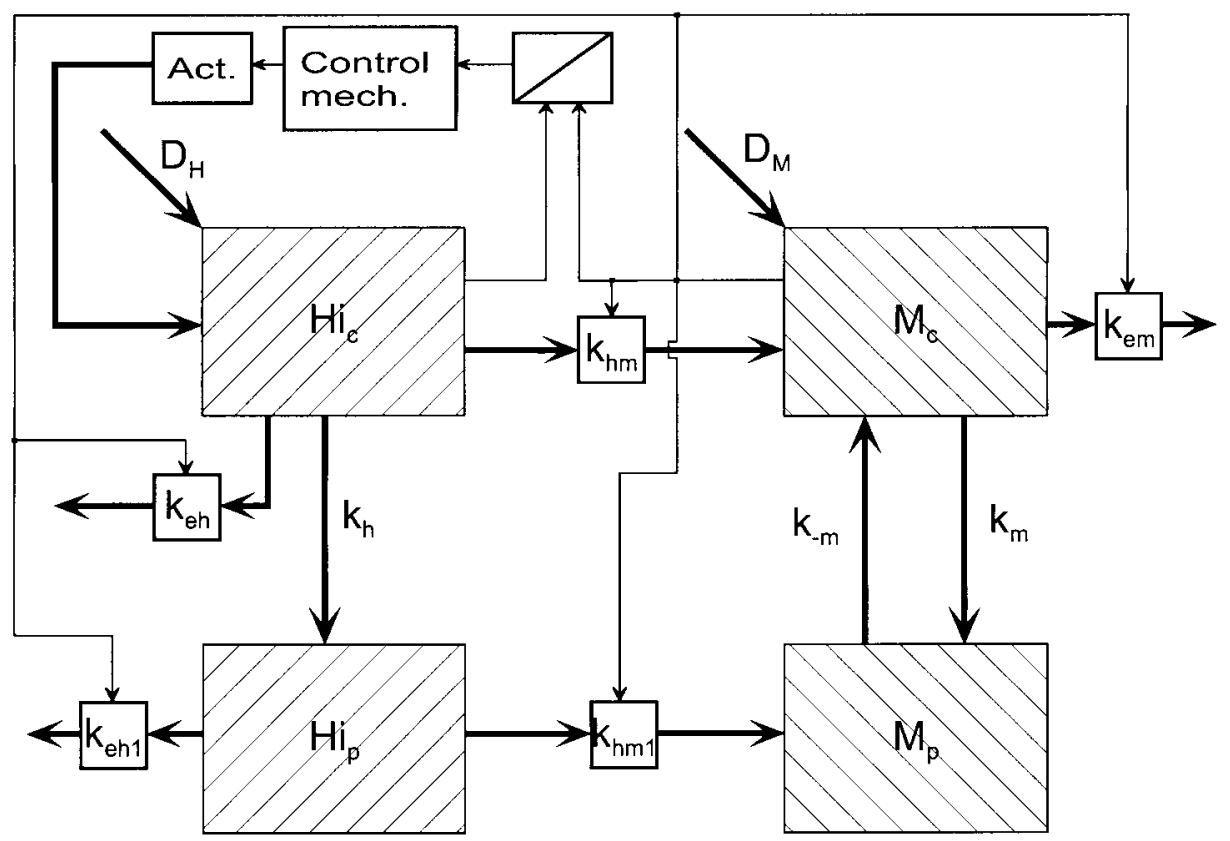

Fig. 9. Model 3. Model of histamine kinetics with control loop for quantity ratio control. In the control loop labels have the following meaning: Control mech.=control algorithm, Act.=generator of rapid endogenous histamine release from mast cells. Note that the rapid endogenous histamine release depends on histamine to methylhistamine quantity ratio as denoted with the thin lines. 
controlling mechanism are herewith identified. Next the control loop structure is defined. The classical ratio control scheme [18,19] is tried with an on/off controller. In this case the two states of the controller are not enough to describe the kinetics, so the controlling algorithm shown in Appendix A (Eqs. (A.10) and (A.11)) is used instead (also Fig. 9). Variable $x$ is an input and represents a difference between desired and current histamine to methylhistamine ratio. Variable $y$ is an output of the controlling algorithm and represents the amount of histamine released from mast cells. $K, D$ and $I$ represent the parameters of the controlling algorithm that are set in the fitting procedure as well as $H i_{e}$, representing constant release of histamine from mast cells. The model with the ratio control, referred to as model 3, explains both types of experiments. However, some minor changes to the model have to be made to achieve an acceptable fit. The experiments present a certain shock to the organism because of the extremely high initial concentrations of both substances. Therefore it is expected that it will take some time, after the concentrations of the substances reach a normal level, before the metabolic and transport processes will also normalize. Therefore the methylation and other metabolic processes are each modelled as step functions with hysteresis (Appendix, Eqs. (A.5)(A.9)). The model 3 (Fig. 9) explains both types of experiments acceptably.

Differential equations for the model 3 are given in Appendix A, the responses of the model are shown in Fig. 10 and the corresponding parameters are summarized in Table 1 . It can be seen that quality of fit is more or less comparable for all models, however only the model 3 has an acceptable physiological background.
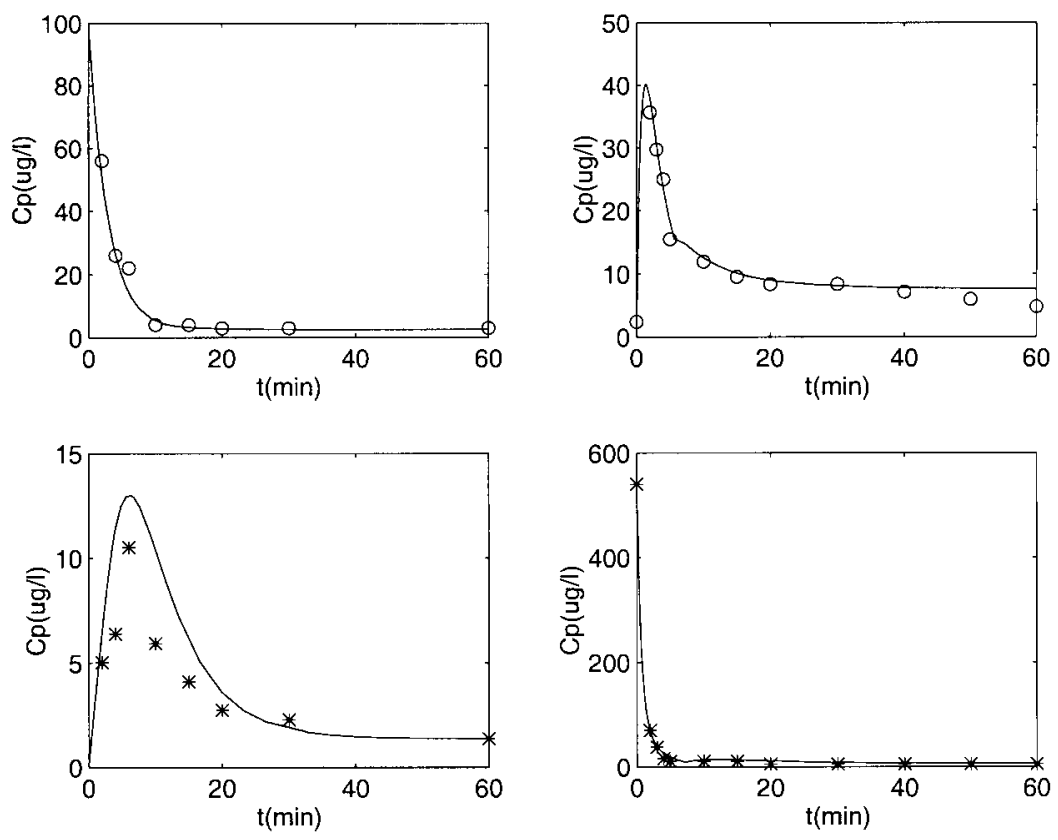

a)

b)

Fig. 10. The model 3 responses (lines) compared to measured data $(\bigcirc=$ histamine, $*=$ methylhistamine) (a) after histamine intravenous administration, (b) after methylhistamine intravenous administration. 


\section{Table 1}

Values of model No. 3 parameters. "Lv1" denotes level at which parameter changes, if the column is empty the parameter is constant. $M$ denotes that parameter is changed when methylhistamine in central compartment reaches certain level. Since the parameters are programmed as step functions with hysteresis, change from high to low value of parameters occurs at different values of $M$ than change from low to high

\begin{tabular}{lll}
\hline Parameter & Value & $L v 1(\mu \mathrm{g})$ \\
\hline$k_{h}\left(\min ^{-1}\right)$ & 0.08 & - \\
$k_{m}\left(\min ^{-1}\right)$ & 0.41 & - \\
$k_{-m}\left(\min ^{-1}\right)$ & 0.52 & - \\
$k_{h m}\left(\min ^{-1}\right)$ & $0.0,0.008$ & $M>2.7, M<1.8$ \\
$k_{h m 1}\left(\min ^{-1}\right)$ & $0.0,0.39$ & $M>2.7, M<1.8$ \\
$k_{e h}\left(\min ^{-1}\right)$ & $0.26,0.05$ & $M>2.7, M<1.8$ \\
$k_{e h 1}\left(\min ^{-1}\right)$ & $0.49,0.08$ & $M>2.7, M<1.8$ \\
$k_{e m}\left(\min ^{-1}\right)$ & $0.94,0.36$ & $M>2.7, M<1.8$ \\
$K(\min$ & & \\
$I(\min )$ & 0.0 & \\
$D$ & 0.0 & \\
$H i_{e}(\mu \mathrm{g} / \mathrm{min})$ & 0.5 & \\
\hline
\end{tabular}

\section{Discussion}

The model fits the available data well enough, considering the amount of the data and the aim of the model (Fig. 10). Next, validation from the physiological side is necessary. The methylhistamine is known to be an inhibitor of histamine- $N$-methyltransferase which controls the methylation process. Therefore the rate of methylation falls when the methylhistamine level rises. The fitting procedures provide model parameters that explain the methylation mechanism in the same manner, as step transitions of parameters $k_{h m}$ and $k_{h m 1}$ go from high to low when rising methylhistamine levels reach a certain level. Furthermore, our experiments show that a rise of methylhistamine above normal levels intensifies the metabolism (elimination) of both amines, which fits well with the model. One can speculate on the basis of these observations that higher levels of methylhistamine inhibit methylation, but yet might speed up oxidative deamination of both methylhistamine itself and the parent molecule histamine by induction of monamine and diamine oxidases. Transport mechanisms from central to peripheral compartment are modelled as diffusion as was found in [16]. This statement is supported by fitting procedures within our study as well. Parameters $k_{h}, k_{-h}, k_{m}$ and $k_{-m}$, which describe transport mechanisms between the compartments, remain constant.

Results obtained during the curve fitting procedure and the model simulation [8] show some interesting facts about the control of histamine to methylhistamine ratio. The outstanding role is played by the additional control loop introduced in model 3. It replaces the time dependent function $\left(0.137 \mu \mathrm{g} / \mathrm{min}+24.9 \mu \mathrm{g} / \mathrm{min} \times e^{-3.1 \times \mathrm{t}}\right)$ of endogenous histamine release in the model $2 \mathrm{~b}$ and makes description of histamine kinetics with only one model for both experiments possible. The control loop is almost inactive, when histamine is injected. This is reasonable since in this case relative changes in ratio are smaller than when methylhistamine is injected. In the second group of experiments, however, the model shows a very rapid release of histamine 
from mast cells. Study of control loop parameters provides the same conclusions. Parameters $K$ and $I$ are negligible opposed to $D$. Such configuration of parameters and the given ratio indicate that the control mechanism is highly sensitive to sudden changes in methylhistamine level. It is an unusual situation for a general control algorithm in technical sciences. However, parameter $I$ is only relevant in the steady state to eliminate the steady state error. There are two reasons why such configuration is possible in our case. Firstly, in the case of biological systems, one can hardly speak of a steady state as it is defined in technical sciences, because of constant significant disturbances from the system itself and the environment. Secondly, survival of an organism more or less depends on how fast, not how exactly, it can adapt to the changes. Therefore, the proposed configuration seems to be logical.

The model presents the kinetics of histamine well enough, considering the original model objectives and the available data. However, some validation on different data sets would contribute a valuable information about this general process.

\section{Conclusion}

The present study of histamine kinetics shows the following important facts:

- Demonstration of interdependence between histamine and methylhistamine by mathematical modelling supports the hypothesis that methylhistamine is a marker of histamine appearance in plasma.

- There is not enough information available to model the processes involving the two substances from basic principles. However, considering the model objective, it is sufficient to build a compartment model based on physiological information and measured data to support the hypothesis.

- Classical linear compartment models are too simple to model the kinetics of the two substances.

- Known facts about histamine and methylhistamine kinetics and known physiological facts can be incorporated in the model.

- Sharp transitions (step functions) are not very likely to take place in nature but can be successfully used as an approximation and an indication of certain changes (considering the objective) or nonlinear behaviour.

- Simulation and modelling results as well as biological experiments support the proposed hypothesis of methylhistamine being a valid marker of histamine appearance in body.

Modelling and simulation is a useful tool in histamine kinetics research and should therefore be used in future studies of histamine kinetics and predictions of histamine profiles from methylhistamine data.

\section{Appendix A. Model 3 equations}

$$
\frac{\mathrm{d} H i_{c}}{\mathrm{~d} t}=y\left(H i_{c}, M_{c}\right)-k_{h m}\left(M_{c}\right) \times H i_{c}-k_{e h}\left(M_{c}\right) \times H i_{c}-k_{h} \times H i_{c}
$$




$$
\begin{aligned}
& \frac{\mathrm{d} M_{c}}{\mathrm{~d} t}=k_{h m}\left(M_{c}\right) \times H i_{c}-k_{e m}\left(M_{c}\right) \times M_{c}-k_{m} \times M_{c}+k_{-m} \times M_{p} \\
& \frac{\mathrm{d} H i_{p}}{\mathrm{~d} t}=k_{h} \times H i_{c}-k_{e h 1}\left(M_{c}\right) \times H i_{p}-k_{h m 1}\left(M_{c}\right) \times H i_{p} \\
& \frac{\mathrm{d} M_{p}}{\mathrm{~d} t}=k_{h m 1}\left(M_{c}\right) \times H i_{p}-k_{-m} \times M_{p}+k_{m} \times M_{c} \\
& k_{h m}\left(M_{c}\right)= \begin{cases}k_{h m}^{\prime} ; & \left(M_{c}<2.7\right) \wedge\left(k_{h m}\left(M_{c}\right)=k_{h m}^{\prime}\right) \\
k_{h m}^{\prime \prime} ; & \left(M_{c}>1.8\right) \wedge\left(k_{h m}\left(M_{c}\right)=k_{h m}^{\prime \prime}\right)\end{cases} \\
& k_{e h}\left(M_{c}\right)= \begin{cases}k_{e h}^{\prime} ; & \left(M_{c}<2.7\right) \wedge\left(k_{e h}\left(M_{c}\right)=k_{e h}^{\prime}\right) \\
k_{e h}^{\prime \prime} ; & \left(M_{c}>1.8\right) \wedge\left(k_{e h}\left(M_{c}\right)=k_{e h}^{\prime \prime}\right)\end{cases} \\
& k_{e m}\left(M_{c}\right)= \begin{cases}k_{e m}^{\prime} ; & \left.M_{c}<2.7\right) \wedge\left(k_{e m}\left(M_{c}\right)=k_{e m}^{\prime}\right) \\
k_{e m}^{\prime \prime} ; & \left(M_{c}>1.8\right) \wedge\left(k_{e m}\left(M_{c}\right)=k_{e m}^{\prime \prime}\right)\end{cases} \\
& k_{h m 1}\left(M_{c}\right)= \begin{cases}k_{h m 1}^{\prime} ; & \left(M_{c}<2.7\right) \wedge\left(k_{h m 1}\left(M_{c}\right)=k_{h m 1}^{\prime}\right) \\
k_{h m 1}^{\prime \prime} ; & \left(M_{c}>1.8\right) \wedge\left(k_{h m 1}\left(M_{c}\right)=k_{h m 1}^{\prime \prime}\right)\end{cases} \\
& k_{e h 1}\left(M_{c}\right)= \begin{cases}k_{e h 1}^{\prime} ; & \left(M_{c}<2.7\right) \wedge\left(k_{e h 1}\left(M_{c}\right)=k_{e h 1}^{\prime}\right) \\
k_{e h 1}^{\prime \prime} ; & \left(M_{c}>1.8\right) \wedge\left(k_{e h 1}\left(M_{c}\right)=k_{e h 1}^{\prime \prime}\right)\end{cases} \\
& x=M_{c} \times 7-H i_{c} \\
& y=K \times x+D \times \frac{\mathrm{d} x}{\mathrm{~d} t}+I \int x \mathrm{~d} t+H i_{e}
\end{aligned}
$$

\section{References}

[1] T. Irman-Florjanc, F. Erjavec, Kinetics of histamine and tele-methylhistamine elimination from rat plasma, Agents and Actions 38 (1993) 76-78.

[2] R. Karba, A. Mrhar, B. Zupančič, M. Atanasijević-Kunc, A. Belič, The role of simulation tool in histamine pharmacokinetical model development, in: T.I. Oren, L.G. Birta (Eds.), Proceedings of the 1995 Summer Computer Simulation Conference, Ottawa, Canada, 1995, pp. 685-688.

[3] G. Maura, A. Vaccari, P.S. Timiros, Effects of chronic stress on the developement of histamine enzyms, Agents and Actions 7 (1977) 437-442.

[4] F.E. Cellier, Continuous System Modelling, Springer-Verlag, New York, 1991.

[5] J.G. Wagner, Pharmacokinetics for the Pharmaceutical Scientist, Technomic Publishing, Lancaster, 1993.

[6] K.R. Godfrey, Compartmental Models and Their Application, Academic Press, London, 1983.

[7] M. Gibaldi, D. Perier, Pharmacokinetics, Marcel Dekker, New York, 1982.

[8] Matko, Karba, Zupančič, Simulation and Modelling of Continuous Systems: A Case Study Approach, Prentice Hall, New York, 1992. 
[9] T. Irman-Florjanc, F. Erjavec, Histamine tele-methylhistamine ratios in rat blood as a function of time after i.v. injection of compound 48/80 or histamine, Agents and Actions 35 (1992) C394-C396 (special conference issue).

[10] J.J. Keyzer, H. Breukelman, B.G. Wolthers, F.J. Richardson, J.G.R. de Monchy, Measurement of N ${ }^{\tau}$-methylhistamine concentrations in plasma and urine as a parameter for histamine release during anaphylactoid reactions, Agents and Actions 16 (1985) 76-79.

[11] Y. Tsuruta, K. Kohashi, Y. Ohkura, Simultaneous determination of histamine and $\mathrm{N}^{\tau}$-methylhistamine in human urine and rat brain by high-performance liquid chromatography with fluorescence detection, J. Chromat. 224 (1981) 105-110.

[12] C. Cobelli, G. Toffolo, Theoretical aspects and practical strategies for the identification of unidentifiable compartmental systems, in: Barker, Young (Eds.), Proceedings of Identification and System Parameter Estimation 1995, York, UK, 1985, pp. 1409-1414.

[13] K.R. Godfrey, J.J. DiStefano, Identifiability of model parameters, in: H.A. Barker, P.C. Young (Eds.), Proceedings of Identification and System Parameter Estimation 1995, York, UK, 1985, pp. 89-113.

[14] P. Eykhoff, System Identification, John Wiley and Sons, London, 1974.

[15] W.A. Ritschel, in: Handbook of Basic Pharmacokinetics, Drug Intelligence Publications Inc, Hamilton, IL, 1992, pp. 161-169.

[16] K.S. Babe, W.E. Serafin, Histamine, Bradykinin and Their Antagonists, in: J.G. Hardman, L.E. Limbirt (Eds.), Goodman and Gilman's. The Pharmacological bases of Therapeutics, McGraw Hill, New York, 1996, p. 583 .

[17] T. Irman-Florjanc, F. Erjavec, The Role of Mast Cells in Redistribution of Tele-methylhistamine in the Body, Agents and Actions 33 (1991) 33-36.

[18] R.C. Dorf, Modern Control Systems, Addison-Wesley, Menlo Park, CA, 1992.

[19] W.S. Levine (Ed.), The Control Handbook, IEEE Press, Boca Raton, FL, 1996.

Aleš Belič was born in 1971. He received his B.Sc. degree from Faculty of Electrical Engineering, University of Ljubljana, Slovenia in 1994. In 1996 he was awarded the Prešeren's award for the students. He is currently a probationary assistant at the Faculty of Electrical Engineering, University of Ljubljana, Slovenia, the main field of his research is modelling and simulation in pharmacokinetics, pharmacodynamics and related sciences.

Iztok Grabnar received his B.Sc. in 1995 from the Faculty of Pharmacy, University of Ljubljana, Slovenia. Currently he is a probationary assistant at the Faculty of Pharmacy, University of Ljubljana, Slovenia, the main fields of his research are modelling and simulation in pharmacokinetics and pharmacodynamics, theory and practice of bioadhesion as well as pharmaceutical informatics.

Rihard Karba received his B.Sc. (1972), M.Sc. (1975) and Ph.D. (1981) degrees from the Faculty of Electrical Engineering, University of Ljubljana, Slovenia. His research interests include modelling and simulation of dynamic systems, as well as modelling and simulation in pharmacokinetics and biomedicine. He is currently a Professor at the Faculty of Electrical Engineering, University of Ljubljana, Slovenia.

Aleš Mrhar received his B.Sc.(1975), M.Sc. (1978) and Ph.D. (1984) degrees from the Faculty of Pharmacy, University of Ljubljana, Slovenia. His research interests include modelling and simulation in pharmacokinetics, design preparation and evaluation of drug delivery systems, theory and practice of bioadhesion as well as pharmaceutical informatics. Currently he is a Professor at the Faculty of Pharmacy, University of Ljubljana, Slovenia.

Tatjana Irman-Florjanc received her Ph.D. (1993) from the Medical Faculty, University of Ljubljana, Slovenia. Her field of research is pharmacokinetics. She is currently an Assistant Professor at Institute of Pharmacology and Experimental Toxicology, Medical Faculty, University of Ljubljana, Slovenia.

Stanislav Primožič received his B.Sc. (1978), M.Sc. (1983) and Ph.D. (1988) degrees from the Faculty of Pharmacy, University of Ljubljana, Slovenia. His research interests include modelling and simulation in pharmacokinetics as well as different disciplines from the field of social pharmacy (pharmacoinformatics, pharmacoepidemiology, communicology). He is currently a Professor at the Faculty of Pharmacy, University of Ljubljana, Slovenia. 